\title{
MODELO DE OTIMIZAÇÃO DA GESTÃO DE RISCO EM EMPRESAS NÃO FINANCEIRAS*
}

\author{
HERBERT KIMURA \\ Professor Doutor da Universidade Presbiteriana Mackenzie - SP \\ E-mail: hkimura@mackenzie.com.br \\ LUIZ CARLOS JACOB PERERA \\ Professor Doutor do Centro Universitário de Franca - SP \\ e da Universidade Presbiteriana Mackenzie - SP \\ E-mail: jperera@mackenzie.com.br
}

\section{RESUMO}

Esta pesquisa apresenta o desenvolvimento de um modelo de otimização da gestão de riscos, através da identificação de uma estratégia de hedge que maximiza a esperança dos lucros. O modelo fundamenta-se na premissa de que fatores de riscos, além de afetar os resultados das empresas, podem, também, estar correlacionados com as oportunidades futuras de investimento. $\mathrm{O}$ modelo proposto incorpora os custos advindos de um endividamento adicional bem como os benefícios da disponibilidade de recursos internos. O trabalho está desenvolvido da seguinte forma: (1) discussão dos aspectos teóricos relacionados ao tema de gestão de riscos no contexto da teoria de finanças; (2) apresentação da metodologia adotada para o desenvolvimento do modelo; (3) derivação dos passos fundamentais que orientaram o modelo matemático, destacando as especificações das variáveis relevantes e os mecanismos de simulação e (4) apresentação dos principais resultados do modelo de hedge ótimo, dentre os quais se destacam: aumento da esperança dos lucros, redução da incerteza em relação aos investimentos, maior estabilidade do nível ótimo de investimento e de endividamento e menor flutuação dos resultados da empresa, decorrente da redução do nível de risco financeiro.

Palavras-chave: Gestão de Riscos; Riscos Financeiros; Hedge Ótimo; Otimização da Gestão de Riscos.

\section{ABSTRACT}

This research presents an optimization model for risk management, through the development of a hedge strategy that maximizes the expected profit of a company. The model is based on the premise that a risk factor, besides affecting the results of the company, can also be correlated with future investment opportunities. The model incorporates the costs of an additional debt as well as the benefits of the availability of internal resources. The work is structured in the following way: (1) discussion of the theoretical aspects related to the theme of risk management in the context of the financial theory; (2) presentation of the methodology adopted for the development of the model; (3) derivation of the fundamental steps that guided the mathematical model, detaching the specifications of the important variables and the simulation mechanisms and (4) discussion of the main results of the hedge model, among which we highlight: increase in profit expectations, reduced uncertainty in relation to the investments, larger stability of investment and debt levels and smaller fluctuation of company results, due to lower financial risk levels.

Keywords: Risk Management; Financial Risks; Optimum Hedge; Optimization of Risk Management. 


\section{INTRODUÇÃO}

A questão da gestão de riscos vem apresentando crescente importância no contexto empresarial. Com o aumento da interdependência dos mercados, as empresas tornam-se mais vulneráveis aos diversos fatores de risco. Aspectos econômicos, financeiros e até mesmo movimentações competitivas propagam-se rapidamente, podendo afetar, consideravelmente, os resultados das empresas.

Supondo que o mercado de capitais seja perfeito e a informação seja simétrica, os teoremas formulados por Modigliani e Miller (1958) podem ser adaptados para demonstrar que a gestão de riscos não é relevante para o valor da empresa. Nessa situação, se as estratégias de investimento são definidas e independentes das decisões de financiamento, a criação de valor para o acionista decorre do aproveitamento adequado das oportunidades de investimento em projetos reais.

Porém, quando se observa o mundo real, algumas premissas de Modigliani e Miller não são obedecidas e, dessa forma, a prática da gestão de riscos pode, efetivamente, gerar valor. Potenciais conflitos de agência, decorrentes de problemas de assimetria de informação, constituem imperfeições de mercado que justificam o uso de mecanismos de gestão de riscos. Sabendo-se que o acesso à informação não é uniforme e supondo-se que os agentes têm disposição a maximizar a própria utilidade em detrimento da riqueza dos principais, a gestão de risco torna-se relevante por sinalizar ao mercado menores problemas de agência.

Flutuações inesperadas de fatores de risco podem levar a níveis de endividamento e liquidez que influenciem o valor da empresa. Em situações de elevada utilização de capital de terceiros, os diversos stakeholders podem penalizar a empresa exigindo maior disclosure de informações, estabelecendo mecanismos de monitoramento e controle e impondo custos advindos da probabilidade de falência em função do nível de endividamento da empresa. Em contrapartida, os stakeholders podem captar sinais positivos e causar um aumento de valor para a empresa. Uma maior liquidez, por exemplo, pode facilitar acesso da empresa a fontes de financiamento caso as disponibilidades internas sejam comprometidas com os projetos de investimentos. Assim, programas de gestão de risco que evitem endividamento excessivo ou que protejam a liquidez das empresas podem ser fontes de geração de riqueza.

Esta pesquisa baseia-se no modelo de gestão de riscos estabelecido por Froot et al. (1993), no qual o endividamento representa capital custoso à empresa. O modelo de Froot et al. (1993) fundamenta-se na premissa de que a gestão de risco é benéfica à medida que flutuações desnecessárias de investimento e endividamento podem ser evitadas. Essas flutuações são decorrentes de um único fator de risco que pode, por exemplo, corresponder ao retorno do produto negociado pela empresa ou ao retorno de um indexador de ativos ou passivos. Avançando o conhecimento teórico sobre gestão de riscos, também serão considerados nesta pesquisa os benefícios associados à liquidez.

O modelo proposto incorpora, portanto, a questão da liquidez através da suposição de que o nível de ativos líquidos, representado pela disponibilidade de recursos internos, pode trazer benefícios em termos de valor. Essa inovação do modelo permite que sejam analisadas, de modo conjunto, praticamente todas as questões financeiras relevantes. O modelo possibilita que sejam avaliadas, diretamente, as decisões de investimento, endividamento, liquidez e gestão de riscos e, indiretamente, a decisão de dividendos.

Assim, no modelo apresentado, a decisão de investimento é levada em consideração através da identificação de que as oportunidades de investimento em projetos são dependentes das realizações do fator de risco. A questão do financiamento está associada à necessidade de capital de terceiros que, juntamente com os recursos gerados internamente, complementa o montante de investimentos que maximiza o lucro da empresa. No modelo, o financiamento externo apresenta custos que diminuem a criação de valor propiciada pelos novos projetos.

O modelo, também, avalia os benefícios advindos da existência de recursos internos que eventualmente podem ser utilizados para os investimentos. Nesse caso, a liquidez da empresa constitui fonte de criação de riqueza. De modo indireto, a questão dos dividendos, também, pode ser contemplada no modelo, pois os recursos gerados internamente representariam fluxos disponíveis para a remuneração do acionista. Finalmente, a questão da gestão de riscos está incorporada no estudo, uma vez que a modelagem possibilita a identificação de um nível de hedge ótimo dos recursos internos que maximize o valor esperado do lucro da empresa.

\section{REFERENCIAL TEÓRICO}

\subsection{Estudos teóricos}

A teoria sobre gestão de riscos fundamenta-se em, praticamente, duas abordagens. A abordagem tradicional para o hedge em corporações não-fi- 
nanceiras tem como origem o trabalho de Stein (1961) que analisa o uso de contratos futuros para a proteção de exposições financeiras. Posteriormente, Ederington (1979) popularizou a abordagem investigando os investimentos em contratos futuros como uma maneira de contrabalancear o risco. A abordagem tradicional supõe que a empresa, por se comportar como um investidor averso a risco, busca a minimização do risco. Estima-se o número ótimo de contratos futuros a serem transacionados em função da minimização do risco da carteira "hedgeada". Segundo premissas simplificadoras, o índice de hedge ótimo é dado pelo quociente da covariância entre as flutuações dos preços à vista e futuro com a variância da flutuação do preço futuro.

A abordagem mais moderna de gestão de riscos leva em consideração os estudos de Jensen e Meckling (1976) sobre as relações contratuais existentes em uma empresa. Devido aos possíveis conflitos de interesse entre administradores, acionistas, credores e demais personagens associados à empresa, a necessidade da gestão de riscos pode depender das relações entre os stakeholders, decorrentes da forma de remuneração, da assimetria de informação, do perfil de risco etc. Os modelos modernos de otimização da função de gestão de riscos envolvem, portanto, a identificação de premissas que regulam as relações de agência ou os conflitos de interesse entre os personagens que participam da administração e do financiamento de uma corporação.

Os modelos tradicionais podem ser considerados casos particulares dos modelos modernos, uma vez que diversos modelos de otimização, que envolvem a identificação de índices de hedge ótimos, baseiam-se, indiretamente, em relações de agência. De acordo com Stein (1961), se a empresa tem comportamento similar a um trader que apresenta aversão a risco e que busca a maximização de sua utilidade, então há um valor ótimo da relação entre a exposição em um contrato futuro e a exposição em uma posição à vista que minimiza o risco da carteira total. A minimização de riscos da empresa pode, portanto, estar associada aos problemas de agência, pois indiretamente reduz a exposição pessoal dos administradores (HAUSHALTER, 2000) devido à maior estabilidade dos fluxos de caixa. Assim, tendo em vista que a probabilidade de surgimento de problemas financeiros com a realização de hedge é consideravelmente baixa, os administradores podem usufruir de maior estabilidade em suas posições, como, por exemplo, através da manutenção de seus empregos. Para o administrador da empresa, essa redução de risco é racional, pois em geral, não possui uma carteira de investimentos muito diversificada (CAMPBELL e KRACAW, 1987) e, portanto, o valor obtido através de seus salários pode representar considerável parcela de sua riqueza.

Heaney e Poitras (1991) obtêm índices ótimos de hedge a partir de dois conjuntos de premissas diferentes. Supondo que os retornos dos preços à vista e dos preços futuros têm distribuição conjunta normal bivariada, os autores derivam os índices de hedge a partir de regressão de mínimos quadrados e de funções de utilidade predeterminadas. Adicionalmente, se houver a possibilidade de captação e aplicação a uma taxa de juros livre de risco, então o índice de hedge ótimo é independente das preferências dos investidores, podendo ser determinado pelos parâmetros da distribuição conjunta. Stulz (1984), por sua vez, estuda o índice ótimo de hedge de uma posição não transacionável nos mercados financeiros através de uma análise intertemporal, supondo que os preços à vista e futuro alteram-se continuamente e que os investidores podem ajustar a todo instante seu comportamento de consumo e hedge.

Myers (2000) compara duas abordagens para a estimação de índices ótimos de hedge que variam com o tempo. Através do estudo de variâncias e covariâncias de commodities agrícolas, o autor estabelece o condicionamento de informação com graus diferentes de sofisticação. Assim, na primeira abordagem são calculadas variâncias e covariâncias amostrais móveis dos erros de predição de preços à vista e preços futuros. Na segunda abordagem, são obtidas estimativas de variâncias e covariâncias através de modelos autoregressivos generalizados de heteroscedasticidade condicional. É identificada forte evidência de que os índices de ótimos hedges variam com o tempo, porém os resultados não mostram melhor performance de modelos mais sofisticados na estimação dos índices de hedges ótimos.

Copeland e Copeland (1999) apresentam um modelo de otimização que representa a maximização do valor da empresa através da avaliação do trade-off entre a redução nos custos esperados de falência e o aumento dos custos esperados do hedge de taxa de câmbio. A partir de uma abordagem baseada em um índice de hedge que minimiza a probabilidade de falência dentro de um intervalo predeterminado de tempo, os autores avaliam um índice entre custo e benefício do hedge, em que o benefício é definido como a redução esperada no custo de falência propiciada pelo hedge e o custo é definido como custo direto do estabelecimento da política de hedge. Stulz (1984) analisa políticas de hedge ótimas sob a premissa de maximização da utilidade esperada da administração, levando-se 
em consideração os impactos das flutuações da taxa de câmbio e dos custos de hedge nas políticas de gestão de risco.

Smith e Stulz (1985) identificam estratégias de hedge em função da forma com que a utilidade esperada do administrador está ligada ao valor da empresa e em função da maneira pela qual os administradores são recompensados. Para evitar a utilização dos fluxos de caixa livres para benefícios não-pecuniários por parte dos administradores, a empresa poderia trocar capital próprio por capital de terceiros criando mecanismos que disciplinam os administradores com relação a suas futuras decisões sobre investimentos. Uma vez que um maior endividamento pode levar a custos de dificuldades financeiras, a gestão de riscos possibilita um maior grau de certeza dos fluxos de caixa dos projetos e, conseqüentemente, a redução dos custos de falência serviria como prevenção contra despesas não-pecuniárias (CULP, 2001). Adicionalmente, se a administração tem informação superior ao acionista no sentido de remoção de variabilidade de resultados através do estabelecimento de políticas de hedge, pode haver incentivos para efetivação dos hedges devido a uma combinação de aversão a risco, mecanismos de contabilização e planos de compensação (DEMARZO e DUFFIE, 1995).

Ross (1996) identifica uma carteira de hedge ótima que possibilita a maximização do valor da empresa, na qual a gestão de riscos possibilita um aumento do índice ótimo de endividamento, fazendo com que a empresa obtenha maiores benefícios fiscais. Nesse caso, a gestão de riscos tem um papel indireto ou secundário, uma vez que o hedge traz incrementos de segunda ordem na riqueza do acionista. No caso, o aumento de valor para o acionista provém, principalmente, da maior possibilidade de alavancagem. Finalmente, Froot et al. (1993) estabelecem uma estrutura analítica na qual a gestão de riscos representa uma interface entre as políticas de investimento e de financiamento. Dessa maneira, o programa de hedge ótimo é função das oportunidades de investimentos disponíveis e da habilidade da empresa em obter financiamento externo. O estudo permite não somente a explicação sobre os motivos para uma empresa realizar o hedge, mas também a identificação da quantidade e do tipo de hedge a ser implementado.

\subsection{Estudos empíricos}

Os estudos empíricos sobre a gestão de riscos em corporações não-financeiras estão associados, principalmente, à investigação sobre a utilização de derivativos, tendo em vista que são instrumentos típicos de redução ou aumento de exposição a riscos. As análises empíricas relacionadas ao uso de derivativos dependem, fortemente, de um amplo levantamento de dados, através do qual, na maioria das vezes, são necessárias avaliações subjetivas dos pesquisadores, principalmente para a caracterização de dados categóricos. Em particular, a identificação de uma empresa usuária ou não-usuária de derivativos pode variar entre diferentes pesquisas, em decorrência da forma de levantamento de dados ou das definições preliminares dos pesquisadores.

Exemplificando, Francis e Stephan (1990) classificam as empresas, com relação ao uso de derivativos, através de pesquisa em bancos de dados financeiros por determinadas palavras-chaves, tais como: "hedging", "swaps" ou "options". Geczy et al. (1997) classificam usuários de derivativos através da busca de referências sobre o uso de derivativos de taxa de câmbio nas demonstrações financeiras de empresas pertencentes ao S\&P 500. Por sua vez, Mian (1996) examina se a empresa utiliza derivativos para gerenciar riscos. Assim, as empresas são classificadas a partir da análise de informações referentes ao uso de derivativos, bem como da identificação de tratamento contábil compatível com operações de hedge.

Dadas as características do processo de coleta de dados, os testes empíricos da teoria de gestão de riscos utilizam, basicamente, duas abordagens diferentes (PETERSEN e TIAGARAJAN, 2000). A primeira abordagem mensura o efeito do uso de derivativos nas exposições financeiras das empresas. A segunda abordagem fundamenta-se na análise comparativa das características de empresas que utilizam e que não utilizam derivativos.

Com relação aos estudos comparativos, Bodnar et al. (1998) observam que o uso de derivativos está associado, positivamente, ao total de faturamento da empresa. Por outro lado, mais da metade dos respondentes da pesquisa, realizada com empresas não-financeiras americanas, alega não utilizar derivativos devido ao nível de risco ser considerado baixo ou poder ser gerenciado de outras formas. Dentre as empresas que usam derivativos, os principais objetivos referem-se à diminuição de riscos e à redução dos custos de financiamento. Poucas empresas utilizam derivativos para fins especulativos, demonstrando, claramente, a disposição da administração em utilizar o mercado de derivativos para protegerem suas exposições a variáveis que são de difícil controle (BODNAR et al., 1998). Nguyen e Faff (2002) identificam que empresas australianas maiores têm maior probabilidade de usar derivativos, em função dos custos de transação. Além disso, quanto maior o endividamento e 
maior o pagamento de dividendos, mais hedge as empresas realizam.

Realizando estudos comparativos, Berkman et al. (1997) observam que as empresas neozelandesas possuem uma gestão de derivativos ainda mais ativa que as empresas americanas, apresentando, também, mecanismos de reportagem do uso de derivativos mais informativos. Uma das motivações para essa evidência reside no fato de as empresas neozelandesas apresentarem maior exposição a risco de moeda e, portanto, maior a preocupação em gerir esse risco. Bodnar e Marston (1996) obtêm, também, evidências de que as empresas em algumas situações realizam hedge com derivativos, porém de modo imperfeito. Em algumas empresas analisadas, posições especulativas parecem ser tomadas como se fossem operações de hedge.

Estudos para a indústria de mineração têm possibilitado análises mais específicas que sobrepujam a avaliação de dados categóricos. Tufano (1996) realiza um estudo detalhado sobre a gestão de riscos em empresas de mineração de ouro. $O$ resultado principal do estudo fundamenta-se no suporte da hipótese de que as empresas utilizam derivativos para a redução de riscos. Existem evidências de que a motivação primária para o estabelecimento de operações de hedge envolve a aversão a risco de acionistas e administradores. No estudo de Tufano (1996), são examinados os determinantes da extensão do hedge executado pela empresa. $O$ autor descobre uma relação fortemente negativa entre a extensão do uso de hedge e a liquidez das companhias de mineração de ouro.

Através da comparação de duas empresas específicas do setor de mineração, com características opostas em relação ao uso de derivativos, Petersen e Thiagaran (2000) identificam que a escolha do método de gerenciamento de riscos, seja através do uso de derivativos sobre ouro, seja através de outras operações, como, por exemplo, empréstimos indexados a ouro, depende das diferenças de habilidade de as empresas ajustarem os custos operacionais e das diferentes necessidades por capital para investimentos. Além disso, os autores, também, identificam que os incentivos da administração em utilizar derivativos para reduzir riscos dependem da forma com que os executivos são recompensados.

Ao estudar evidências de produtores de óleo e de gás, Haushalter (2000) identifica a relação entre hedge e custos de captação. Dessa maneira, o autor observa que a probabilidade de realizar hedge está associada às economias de escala dos custos de hedging e ao risco de base dos instrumentos de proteção. Companhias mais propensas à ad- ministração de riscos são maiores e têm produção proveniente, principalmente, de regiões nas quais o produto extraído tem alta correlação com ativos objetos aos quais derivativos negociados em Bolsa estão vinculados.

Howton e Perfect (1998) relatam que as empresas têm menor probabilidade de utilizar contratos a termo e futuros para gerir risco de taxa de câmbio quando sua liquidez é elevada e quando dificuldades financeiras não constituem ameaça potencial. Além disso, a probabilidade de uso de derivativos é baixa quando o índice de despesas em pesquisa e desenvolvimento com relação às vendas é baixo, corroborando resultados de Nance et al. (1993) e Dolde (1995). Cohen et al. (2000), através de pesquisa com executivos financeiros, examinam se a estrutura dos contratos de remuneração dos administradores afeta a forma de gestão de risco das empresas. Nesse sentido, o estudo sugere que diferenças no nível de exposição a risco das empresas estão associadas ao volume de opções sobre ações e demais compensações diferidas utilizadas pelas empresas como incentivos aos executivos.

É importante destacar que estudos empíricos têm demonstrado um certo distanciamento entre a teoria baseada em um hedge ótimo e a prática em gestão de riscos. Guay e Kothari (2003), em uma amostra de 234 grandes empresas não-financeiras americanas, identificam que o índice de hedge é extremamente inferior ao valor financeiro de exposição a riscos. Moosa (2004), através de um estudo de três estratégias distintas, sugere que o hedge do risco de taxa de câmbio através de derivativos não conduziria, no longo prazo, a resultados superiores à manutenção da exposição.

\section{METODOLOGIA}

No referencial teórico foram apresentadas e discutidas as abordagens sobre gestão de riscos visando fundamentar a importância do modelo proposto. A partir da identificação de variáveis relevantes para a valoração de empresas, foi desenvolvido um modelo quantitativo, no qual buscou-se avaliar qual o nível de hedge que maximiza os lucros esperados da empresa. Aprimorou-se o modelo desenvolvido por Froot et al. (1993), através da análise integrada da gestão de riscos, das oportunidades de investimento, das necessidades de endividamento e da disponibilidade de recursos internos. A introdução da variável liquidez, aspecto financeiro relevante para a gestão de riscos, torna o modelo mais completo e traz, também, maior complexidade nas derivações matemáticas. A partir da formulação do nível de hedge ótimo, foram simulados os possíveis 
resultados de empresas "hedgeadas" e de empresas não-"hedgeadas" para evidenciar os ganhos proporcionados pelas estratégias de gestão de riscos.

\subsection{Desenvolvimento do modelo}

Conforme já discutido anteriormente, segundo determinadas premissas análogas às de Modigliani e Miller (1958), a decisão de endividamento, a política de dividendo, a administração da liquidez e a gestão de riscos pouco contribuem para o processo de criação de valor ao acionista. Nessas condições, apenas a decisão de investimento seria relevante. Porém, quando violações às premissas são encontradas, modelos financeiros mais sofisticados, que relaxam diversas suposições, podem trazer subsídios para a prática financeira, justificando a importância dos diversos aspectos de administração financeira.

No contexto discutido neste trabalho, a função lucro $\mathrm{P}$ pode ser definida como o valor presente líquido $\mathrm{F}$ das oportunidades de investimentos, subtraído dos custos $\mathrm{C}$ provenientes do endividamento necessário para a implementação dos projetos e adicionado pelo benefício $B$ da liquidez advindo da existência de recursos internos que podem financiar parte dos investimentos, ou seja,

$$
P(w)=F(I)-C(e)+B(w) \text {, em que } I=e+w
$$

sendo que:

$I$ = representa o nível de investimento;

$e=$ está associado ao financiamento adicional;

$w=$ corresponde aos fluxos gerados internamente.

Assim, o investimento em projetos é financiado por recursos internos (w) e endividamento com capital de terceiros (e). Supondo que o fator de risco ao qual a empresa está exposta influencia as oportunidades de investimento, então o valor presente líquido pode ser estabelecido pela equação a seguir:

$$
F(I)=\theta f(I)-I, \operatorname{com} \theta=\rho(\varepsilon-1)+1,
$$

sendo que:

$\rho=$ uma medida da correlação entre as oportunidades de investimento e o fator de risco e

$\varepsilon=0$ retorno do fator de risco que influencia o resultado da empresa; para fins de simplicidade matemática, será considerado que o fator de risco possui distribuição normal com média 1 e variância $\sigma^{2}$.

No modelo proposto, $f(l)$ será definida como a função que associa, para cada montante de investi- mento I, um valor presente dos novos projetos. Tendo em vista que as oportunidades de investimento viáveis se esgotam em função de restrições específicas das empresas, como, por exemplo, tecnologia, competição, será considerado que:

$$
\frac{d f(I)}{d I}=f_{I}>0 \text { e } \frac{d f_{I}}{d I}=f_{I I}<0
$$

Ou seja, a criação de valor dos investimentos é crescente, porém investimentos adicionais conduzem a incrementos cada vez menores no valor presente dos projetos.

É importante observar, conforme já discutido anteriormente, que o valor presente líquido dos projetos é influenciado pelo fator de risco $\varepsilon$. Caso a correlação $\rho$ seja positiva, então situações em que o fator de risco supera seu valor esperado aumentam os valores presentes das oportunidades de investimento. Quando o fator de risco realizado fica abaixo de seu valor médio, as oportunidades de investimento tendem a perder valor. $O$ caso em que $\rho$ é maior que zero pode ser exemplificado por uma empresa exportadora, sujeita ao risco de flutuações de taxa de câmbio. Assim, um aumento do dólar poderia influenciar positivamente as possibilidades de negócio, fazendo com que os projetos potenciais conduzissem a maiores valores presentes. Na situação em que a correlação $\rho$ é negativa, se o valor realizado do fator de risco for menor que sua média, indicando um resultado abaixo do esperado, então as oportunidades de investimento envolvem maiores valores presentes. Na situação oposta, se a realização do fator de risco for maior que o valor esperado, as oportunidades de investimento conduzem a menores valores presentes. Um exemplo para o caso de correlação negativa pode ser representado por uma empresa exposta ao risco de taxa de câmbio, na qual uma queda na taxa de câmbio diminui os fluxos de caixa gerados internamente, mas ao mesmo tempo, implica melhores oportunidades de investimento para o futuro.

Os custos decorrentes do endividamento podem ter várias origens. De acordo com Froot et al. (1993), esses custos podem advir de perdas de valor associadas a um incremento das dificuldades financeiras e a um aumento do potencial de falência da empresa. Assim, o maior endividamento pode acarretar custos diretos na forma de pagamento de serviços legais em caso de falência e custos indiretos na forma de decréscimo na competitividade da empresa e de ineficiência operacional que podem surgir, por exemplo, com a questão do sub-investimento. Além disso, a assimetria de informações 
entre administradores e investidores externos pode, também, implicar custos de endividamento. Empresas com oportunidades de investimento adequadas, porém com nível de endividamento considerado insatisfatório, podem ter seu valor diminuído pelo mercado, devido à diferença informacional. Problemas de agência, igualmente, podem conduzir a custos do endividamento. Por exemplo, os acionistas incorrem em desembolsos para monitorar o comportamento dos administradores com relação ao nível de dívidas. Adicionalmente, os gestores podem obter ganhos particulares através da limitação do capital de terceiros, trabalhando em patamares de endividamento não-otimizados.

Considerando que um endividamento excessivo pode aumentar, consideravelmente, os custos de falência, então a função $C(e)$ é modelada da seguinte forma:

$$
\frac{d C(e)}{d e}=C_{e}>0 \mathrm{e} \frac{d C_{e}}{d e}=\mathrm{C}_{e e}>0
$$

Com isso, os custos de endividamento são crescentes e incrementos de endividamento causam incrementos de custos cada vez maiores.

O benefício da liquidez tem fundamentação na tentativa de diminuição da assimetria de informação. A sinalização através da utilização de recursos próprios para a realização de investimentos confere maior segurança aos investidores externos. Dessa forma, o uso de recursos internos sinaliza comprometimento do capital próprio com os projetos, diminuindo a penalidade imposta pelo mercado decorrente da necessidade de um maior endividamento. Conflitos de agência entre acionistas e credores, também, são amenizados por uma maior liquidez da empresa, pois o direcionamento de recursos internos para investimentos pode sugerir que a implementação de projetos gere mais riqueza que a distribuição de dividendos aos acionistas.

Supondo que acima de um certo limite a liquidez traga benefícios marginais desprezíveis para a empresa, então condições razoáveis para $B(w)$ podem ser descritas por:

$$
\frac{d B(w)}{d w}=B_{w}>0 \text { e } \frac{d B_{w}}{d w}=B_{w w}>0
$$

É importante observar que a modelagem exclui potenciais penalidades impostas à empresa pelo excesso de liquidez, que poderia sinalizar falta de oportunidades de investimentos ou níveis excessivos de conservadorismo por parte dos administradores.
Tendo sido definidas as funções relevantes, o próximo passo é estabelecer a dinâmica financeira. O modelo proposto consiste na análise de três instantes: $t_{0}, t_{1}$ e $t_{2}$. Iniciando a avaliação a partir do último período, pode-se identificar a relevância da estratégia de gestão de riscos a ser definida no período inicial. Assim, em $t_{2}$ a empresa obtém um resultado que é função do nível ótimo de investimento realizado em $t_{1}$. O investimento estabelecido em $t_{1}$, juntamente com a disponibilidade de recursos internos da empresa nesse período, define o endividamento adicional necessário.

Considerando que realizações do fator de risco podem alterar os ativos líquidos da empresa que serão utilizados para investimentos, deve-se em $t_{0}$ identificar qual fração das disponibilidades internas iniciais deve ser protegida. Portanto, a decisão de hedge ocorre em $t_{0}$, implicando que o mecanismo de otimização seja fundamentado no valor esperado ou na esperança da função lucro, devido à incerteza associada ao fator de risco no instante inicial.

Seguindo a dinâmica descrita, em $t_{1}$ a empresa deve identificar o nível de investimento que maximiza o lucro. Assim, o problema de otimização é dado por:

$$
\max P(w)
$$

Derivando a função lucro em relação ao investimento, tem-se:

$$
\frac{d P}{d I}=\frac{d(\theta f(I)-I)}{d I}-\frac{\partial C(e)}{\partial e} \frac{\partial e}{\partial I}+\frac{\partial B}{\partial w} \frac{\partial w}{\partial I}
$$

Uma vez que no instante $t_{1}$ o fator de risco já foi realizado e, portanto, pode ser observado, então w e $\theta$ são determinados, ou seja, sendo $w$ fixo, as variações no investimento são totalmente financiadas por variações no nível de endividamento. Portanto, as condições de primeira e segunda ordem estabelecem que o nível ótimo de investimento é dado por:

$$
\begin{aligned}
& \frac{d P}{d I}=0 \Rightarrow \theta f_{I}-1-C_{e}=0 \mathrm{e} \\
& \frac{d^{2} P}{d I^{2}}<0 \Rightarrow \theta f_{I I}-C_{e e}=0
\end{aligned}
$$

Conforme discutida anteriormente, a questão do hedge surge em $t_{0}$, pois o valor dos recursos internos que estarão disponíveis em $\mathrm{t}_{1}$ está associado ao fator de risco $\varepsilon$. No modelo, em $t_{0}$ a empresa dispõe de recursos $w_{0}$ que estão sujeitos a flutuações de valor em função do fator de risco. A empresa 
pode escolher em $t_{0}$ realizar um hedge de $\mathrm{w}_{0}$ de tal forma a eliminar parte da incerteza dos recursos internos $w$ que serão utilizados em $t_{1}$ para financiar $o$ investimento ótimo. Assim, a decisão de gestão de riscos envolverá o estabelecimento da porcentagem $h$ dos recursos internos $w_{0}$ que será protegida contra flutuações do fator de risco $\varepsilon$.

Considerando o fator de risco $\varepsilon$, os recursos internos $w$ em $t_{1}$ podem ser modelados por:

$$
w=w_{0}(h+(1-h) \varepsilon)
$$

em que:

$$
\begin{aligned}
w_{0} \neq 0= & \text { representa o montante de recursos inter- } \\
& \text { nos disponíveis em } \mathrm{t}_{0} \mathrm{e} \\
h \quad & \text { representa o índice de hedge. }
\end{aligned}
$$

É fácil identificar dois casos especiais. Se $h=1$, ou seja, se o hedge for completo, $w=w_{0}$ e, dessa forma, não há incerteza com relação aos recursos internos disponíveis em $t_{1}$. Se $h=0$, então $w=w_{0} \varepsilon$ $e$, portanto, os recursos internos em $t_{1}$ refletirão toda a possível variabilidade do fator de risco $\varepsilon$. Dependendo da forma e dos parâmetros da função de valor presente dos investimentos, da função de custos associados ao endividamento e da função de benefícios da liquidez, o nível de hedge ótimo pode ser diferente desses casos especiais. Mais ainda, em uma análise preliminar, considerando os possíveis valores da correlação $\rho$ entre o fator de risco e as oportunidades de investimento, não se pode descartar a possibilidade de que $\mathrm{h}$ tenha valores fora do intervalo $[0,1]$. Valores maiores do que a unidade refletem que o hedge é feito sobre um montante superior aos recursos internos inicialmente disponíveis. Valores negativos implicam que a gestão de riscos deixa os recursos internos mais vulneráveis às flutuações do fator de risco. Nesse caso, a gestão de riscos sugere um aumento de exposição ao risco, para que se aproveite melhor as oportunidades de investimento.

Considerando que em $\mathrm{t}_{0} \varepsilon$ ainda não é definido, para a obtenção da fórmula do hedge ótimo, deve-se resolver o problema de otimização, identificando-se $h^{*}$ tal que a esperança da função lucro seja máxima. Com isso, o problema de otimização é dado por:

$$
\max _{h} E_{0}[P(w(\varepsilon, h))]
$$

Assim, em função da aleatoriedade do fator de risco, será maximizado o valor esperado da função lucro, medida no instante $t_{0}$. Considerando as premissas do modelo, a maximização envolve a seguinte condição de primeira ordem:

$$
\frac{d E_{0}[P(w(\varepsilon, h))]}{d h}=0
$$

Rubinstein (1976) obtém uma propriedade útil para o problema em questão. Sendo x e y variáveis aleatórias com distribuição normal bivariada e $\mathrm{g}(\mathrm{y})$ uma função diferenciável em relação a y, com $|g(y)| \leq k<\infty$, então $\operatorname{Cov}[x, g(y)]=E\left[g^{\prime}(y)\right] \operatorname{Cov}(x, y)$. Utilizando o teorema, sendo $x$ e y são iguais a $\varepsilon$ e, considerando que a derivada do lucro em relação aos recursos internos é também derivável em relação a $\varepsilon$, pode-se demonstrar que a condição de primeira ordem pode ser descrita por:

$$
E_{0}\left[\frac{d\left(\frac{\partial P}{\partial w}\right)}{d \varepsilon}\right]=0
$$

Resolvendo a equação acima, demonstra-se que:

$$
\frac{d\left(\frac{\partial P}{\partial w}\right)}{d \varepsilon}=\rho f_{I} \frac{\partial I}{\partial w}+\theta \frac{\partial f_{I}}{\partial I} \frac{\partial w}{\partial \varepsilon}\left(\frac{\partial I}{\partial w}\right)^{2}+\left(\theta f_{I}-1\right) \frac{\partial^{2} I}{\partial w^{2}} \frac{\partial w}{\partial \varepsilon}-C_{e e} \frac{\partial w}{\partial \varepsilon}\left(\frac{\partial e}{\partial w}\right)^{2}-C_{e} \frac{\partial^{2} e}{\partial w^{2}} \frac{\partial w}{\partial \varepsilon}+B_{w w} \frac{\partial w}{\partial \varepsilon}
$$

Finalmente, utilizando o teorema da função implícita, através de manipulações matemáticas obtém-se o resultado do nível de hedge que maximiza o lucro esperado da empresa.

$$
h^{*}=1+\frac{\rho}{w_{0}} \frac{E_{0}\left[-\frac{f_{I} C_{e e}}{\theta f_{I I}-C_{e e}}\right]}{E_{0}\left[-\frac{\theta f_{I I} C_{e e}}{\theta f_{I I}-C_{e e}}+B_{w w}\right]}
$$

\subsection{Simulação do modelo}

A fórmula analítica dada pela equação 14 possibilita que seja identificado o nível de hedge ótimo para maximização do lucro esperado. No modelo, as oportunidades de investimento variam em função de um fator de risco que afeta, também, as disponibilidades de recursos internos. A estratégia de gestão de riscos envolve, portanto, a definição de um índice 
de hedge das disponibilidades que maximize o lucro esperado da empresa, considerando, de modo conjunto, o valor presente dos investimentos, os custos do endividamento e os benefícios da liquidez.

Uma vez que a fórmula do hedge ótimo é genérica e complexa, torna-se difícil avaliar como as diversas características associadas à empresa podem influenciar a decisão de administração de riscos. A simulação realizada nessa seção possibilita, através do estabelecimento de funções específicas, a avaliação da sensibilidade da estratégia de hedge a alterações dos diferentes fatores relevantes para a gestão de riscos.

Será investigado o comportamento dos mecanismos ótimos de gestão de riscos considerando-se funções do tipo Cobb-Douglas para o cálculo das variáveis das quais a função de lucro esperado depende. Essas funções específicas foram escolhidas pela facilidade de tratamento matemático e pela flexibilidade na construção de modelos que apresentem comportamento útil e realista. Spano (2001), também, utiliza funções Cobb-Douglas obtendo, através da expansão de Taylor de segunda ordem, uma fórmula analítica aproximada para o hedge ótimo, no caso em que inexiste o benefício da liquidez. Nesta pesquisa, ao invés de se procurarem resultados de valor esperado aproximados analiticamente, serão simuladas diversas realizações do fator de risco, de modo que as esperanças da equação 14 possam ser estimadas através das médias amostrais dos valores das variáveis aleatórias.

A equação 14 representa o índice de hedge que maximiza a esperança da função lucro. As funções $f(l), C(e)$ e $B(w)$ devem ser tais que as condições de primeira e segunda ordem, descritas pela equação 8 possam ser verificadas. $O$ resultado do hedge ótimo deve obedecer ao sistema definido pelas equações 1, 8 e 14 .

Para as simulações, será definida, inicialmente, a função que representa os valores presentes dos projetos de investimento:

$$
f(I)=\frac{\phi_{l} I^{1-\beta_{l}}}{1-\beta_{1}}
$$

em que $\phi_{1}$ e $\beta_{1}$ são constantes, tais que $\phi_{1}>0$ é um fator que ajusta a função de valor presente à escala de oportunidades de negócios da empresa; $0<\beta_{1}<1$ é um parâmetro associado à elasticidade do valor presente das oportunidades de negócio em relação à decisão de investimentos.

De fato, $1-\beta_{1}$ representa um conceito de elasticidade, pois:

$$
\frac{\frac{d f(I)}{f(I)}}{\frac{d I}{I}}=\frac{d f(I)}{d I} \frac{I}{f(I)}=\phi_{I} I^{-\beta_{l}} \frac{I}{\frac{\phi_{I} I^{I-\beta_{l}}}{1-\beta_{1}}}=1-\beta_{I}
$$

Além disso, a função custo pode ser definida como:

$$
C(e)=\frac{\phi_{2} e^{1+\beta_{2}}}{1+\beta_{2}}
$$

em que $\phi_{2}$ e $\beta_{2}$ são constantes, tais que $\phi_{2}>0$ representa um fator de escala que corrige os custos do endividamento com capital de terceiros às características da empresa; $0<\beta_{2}<1$ está associado à elasticidade do custo em relação à quantidade de dinheiro emprestado.

Finalmente, os benefícios advindos da liquidez da empresa são definidos como:

$$
B(w)=\frac{\phi_{3} w^{1-\beta_{3}}}{1-\beta_{3}}
$$

em que $\phi_{3}$ e $\beta_{3}$ são tais que $\phi_{3}>0$ é um fator de escala que possibilita ajustar o nível de benefício da liquidez para tipos diferentes de empresas ou para condições diferenciadas de mercado; $0<\beta_{3}<1$ é um parâmetro representativo da elasticidade do benefício da liquidez em relação à disponibilidade dos recursos internos.

Essa especificação das funções $f(l), C(e)$ e $B(w)$ permite a definição do sistema de equações que deve ser observado. No caso da empresa não "hedgeada", o nível de investimento ótimo deve satisfazer à condição de primeira ordem da equação 8 e a relação de equilíbrio entre investimentos e fontes de financiamento apresentada na equação 1 . Considerando-se as condições impostas no modelo de simulação, deve-se ter, simultaneamente, para a empresa não "hedgeada":

$$
\theta \phi_{1} I^{-\beta_{1}}-1-\phi_{2} e^{\beta_{2}}=0 \text { e } I=e+w_{0} \varepsilon
$$

Por sua vez, a empresa "hedgeada" deve, além de satisfazer à condição de primeira ordem dada na equação 19, obedecer à equação de equilíbrio dos investimentos e das fontes de recursos e à equação de hedge ótimo. A equação de equilíbrio é dada por:

$$
I=e+w_{0}(h+(1-h) \varepsilon)
$$

Já a equação de hedge ótimo, para o caso das funções Cobb-Douglas, pode ser obtida substituin- 
do-se os resultados das derivadas das funções $f(l)$, $\mathrm{C}(\mathrm{e})$ e $\mathrm{B}(\mathrm{w})$ na equação 14:

$$
h^{*}=1+\frac{\rho}{w_{0}} \frac{E_{0}\left[\frac{\phi_{1} \phi_{2} \beta_{2} I^{-\beta_{1}} e^{\beta_{2}-1}}{\theta \phi_{1} \beta_{1} I^{-1-\beta_{l}}+\phi_{2} \beta_{2} e^{\beta_{2}-1}}\right]}{E_{0}\left[\frac{-\theta \phi_{1} \phi_{2} \beta_{1} \beta_{2} I^{-\beta_{1}-1} e^{\beta_{2}-1}}{\theta \phi_{1} \beta_{1} I^{-1-\beta_{1}}+\phi_{2} \beta_{2} e^{\beta_{2}-1}}-\phi_{3} \beta_{3} w^{-\beta_{3}-1}\right]}
$$

Assim, em termos práticos, a estimativa do hedge ótimo é obtida através de um procedimento recursivo baseado em um algoritmo computacional que segue os seguintes passos: (1) geração das realizações do fator de risco, (2) definição dos parâmetros das funções e da semente do processo recursivo, (3) cálculo do nível ótimo de investimentos conforme equação 8, (4) cálculo do nível de endividamento e do nível de recursos internos, (5) cálculo de parâmetros auxiliares representativos das esperanças da equação 21 , (6) iteração do nível de hedge dado pela equação 21 , (7) verificação do critério de parada que define a necessidade de iterações adicionais para refinamento da estimativa do hedge ótimo. No procedimento, são simuladas realizações do fator de risco e as esperanças são obtidas através das médias amostrais das realizações. Além disso, mecanismos de cálculo numérico são utilizados para a identificação de raízes de equações não-lineares.

\section{AVALIAÇÃO DOS RESULTADOS}

Para a simulação, foram identificados parâmetros das funções de valor presente, de custo do endividamento e de benefício da liquidez que conduzissem a valores com ordem de grandeza semelhante e que permitissem análises comparativas relevantes. Além disso, visando a uma maior adequação à realidade, os parâmetros implicam que as oportunidades de investimento são mais importantes que os custos e os benefícios considerados na modelagem. A Tabela 1 mostra os parâmetros utilizados na simulação realizada inicialmente para investigação do impacto do hedge no resultado da empresa, considerando-se diferentes realizações do fator de risco. Nessa simulação, estabeleceu-se que a correlação entre o fator de risco e as oportunidades de investimento é positiva.

Sob as condições definidas na tabela anterior, obtiveram-se os resultados, descritos no Gráfico 1, do volume de investimento ótimo em função das diferentes realizações do fator de risco. São estudados dois casos: no primeiro caso, considerou-se
Tabela 1 - Parâmetros do modelo de simulação para análise dos níveis de lucro e das necessidades de investimento

\begin{tabular}{lcc} 
Variável & Parâmetro & Valor \\
Fator de risco $(\varepsilon)$ & $\mu$ & 1 \\
& $\sigma$ & 0,2 \\
& $\rho$ & 0,1 \\
\hline Valor presente: $\mathrm{f}(\mathrm{l})$ & $\beta_{1}$ & 0,25 \\
& $\phi_{1}$ & 20 \\
\hline Custo do endividamento: $\mathrm{C}(\mathrm{e})$ & $\beta_{2}$ & 0,5 \\
& $\phi_{2}$ & 2 \\
\hline Benefício da liquidez: $\mathrm{B}(\mathrm{w})$ & $\beta_{3}$ & 0,1 \\
& $\phi_{3}$ & 0,2 \\
\hline Recursos internos $(\mathrm{w})$ & $\mathrm{w}_{0}$ & 50 \\
\hline Valores iniciais & $\mathrm{I}_{0}$ & 20 \\
& $\mathrm{~h}_{0}$ & 0,5
\end{tabular}

Fonte: elaborada pelos autores.

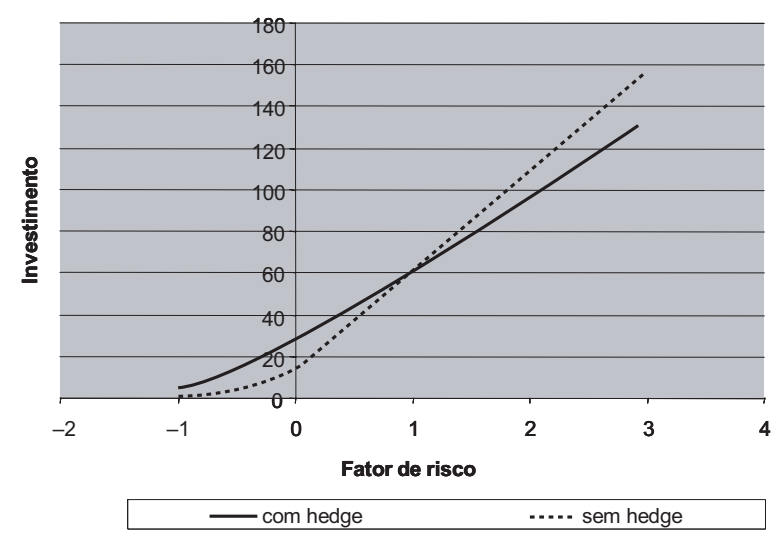

Fonte: Elaborado pelos autores.

\section{Gráfico 1 - Investimento ótimo sob diferentes realizações do fator de risco}

que a empresa adota uma estratégia otimizada de hedge e, no segundo, considerou-se que a empresa não estabelece nenhum programa de gestão de riscos. É importante observar que o hedge suaviza o comportamento do nível de investimento ótimo. O menor intervalo de variação para o caso da empresa "hedgeada" mostra que a gestão de riscos implica menor incerteza associada ao total de investimentos que serão necessários para o aproveitamento das oportunidades de negócio que, por sua vez, dependem das realizações do fator de risco. 
O financiamento dos investimentos ótimos é realizado através do uso de recursos internos e de endividamento externo. O Gráfico 2 mostra que a empresa "hedgeada" apresenta menores variações de recursos internos, refletindo a maior estabilidade do nível ótimo de investimento.

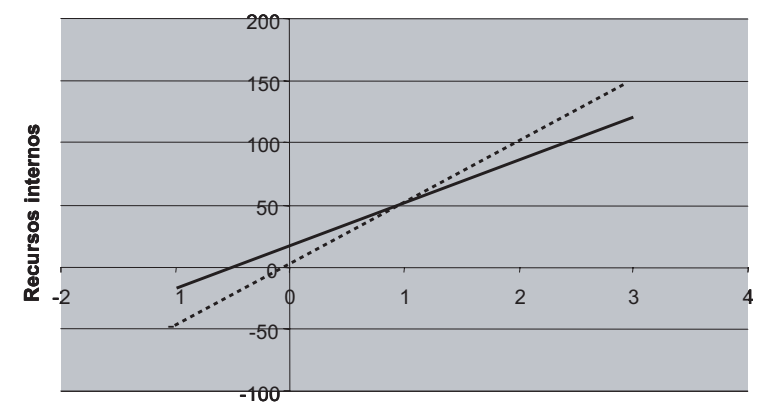

Fator de risco

Fonte: Elaborado pelos autores.

\section{Gráfico 2 - Recursos internos sob diferentes realizações do fator de risco}

De modo análogo, o endividamento externo também apresenta menores variações para a empresa que realiza o hedge.

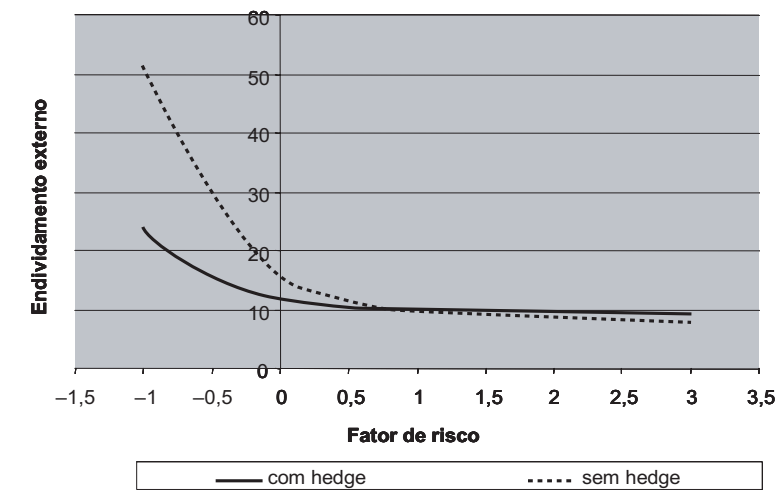

Fonte: Elaborado pelos autores.

\section{Gráfico 3 - Endividamento externo sob diferentes realizações do fator de risco}

Uma observação importante refere-se ao comportamento do nível de dívida que varia com as realizações do fator de risco. O endividamento é parte integrante tanto da condição de primeira ordem dada pela equação 19 quanto do lucro esperado da empresa e, portanto, seu impacto no modelo de otimização é extremamente relevante. Na simulação efetuada, observa-se que, a partir de determinado nível de investimentos necessários, a empresa deve privilegiar o uso de recursos disponíveis internamente, uma vez que a dívida representa fonte de destruição de lucro. Apesar de o hedge implicar, para realizações elevadas do fator de risco, maiores custos de dívida, ele conduz, também, a um lucro esperado maior, pois realizações baixas do fator de risco exigem, para a empresa não "hedgeada", endividamento bem superior ao da empresa "hedgeada".

Finalmente, os lucros das empresas "hedgeadas" e das não "hedgeadas" podem ser comparados através do Gráfico 4. Pode-se observar que o hedge diminui o intervalo de variação do lucro, suavizando o impacto das realizações dos fatores de risco. Em termos de valores esperados, para a empresa "hedgeada", o menor lucro em realizações elevadas do fator de risco é mais do que compensado pelo maior lucro em realizações baixas do fator de risco.

Assim, as simulações numéricas confirmam os resultados esperados: o hedge estabiliza as necessidades de investimento e ajusta as disponibilidades de recursos internos de tal maneira que as exigências de endividamento têm um custo médio menor. $O$ benefício da liquidez contribui para uma maior necessidade de hedge, porém não é fator decisivo para distinguir as funções de lucro, pois as inclinações das curvas de recursos internos com o fator de risco são semelhantes para as estratégias com e sem hedge.

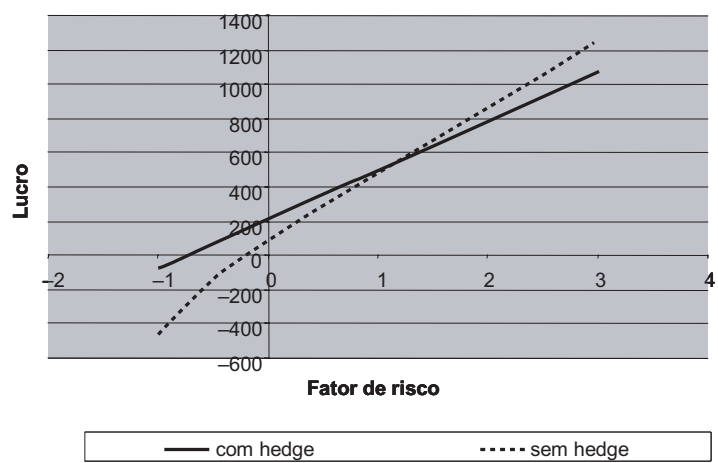

Fonte: Elaborado pelos autores.

\section{Gráfico 4 - Lucro sob diferentes realizações do fator de risco}

No estudo discutido, foram apresentados os resultados da simulação utilizando-se uma correlação positiva entre o fator de risco e as oportunidades de investimento. Diversas outras simulações podem 
ser, também, avaliadas alterando-se os parâmetros da Tabela 1, como por exemplo, a avaliação do nível ótimo de hedge em função da correlação do fator de risco com as oportunidades de investimentos.

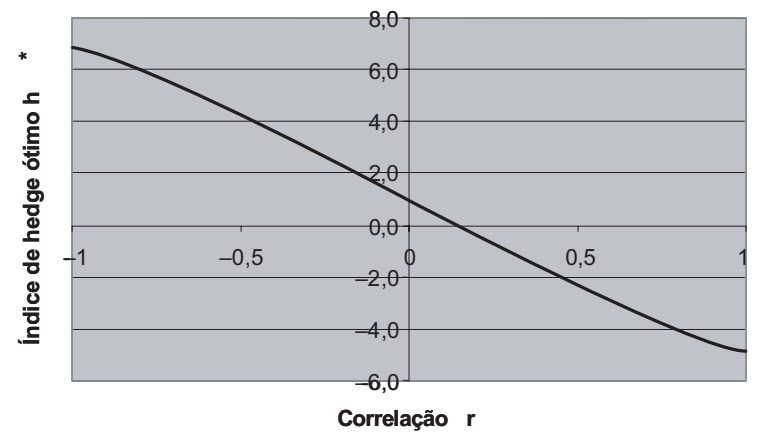

Fonte: Elaborado pelos autores.

\section{Gráfico 5 - Índice de hedge ótimo em função da correlação entre as oportunidades de investimento e o fator de risco}

É importante ressaltar que o hedge ótimo não é função linear da correlação. Apesar de a equação 14 aparentar descrever uma relação linear, não se pode esquecer que o parâmetro $\theta$ também é função da correlação $\rho$. O nível de eliminação ou assunção de riscos que maximiza o lucro esperado depende dos ganhos propiciados pelos investimentos e também dos custos do endividamento e dos benefícios da liquidez. Assim, o comportamento do hedge ótimo é sensível não somente aos parâmetros da função $f$ e à correlação $\rho$, como também aos parâmetros das funções $\mathrm{C}$ e $\mathrm{B}$ e aos recursos iniciais disponíveis $\mathrm{w}_{0}$, conforme a equação 14 determina. Variando as disponibilidades a serem geridas em $t_{0}$, pode-se encontrar o comportamento qualitativo do índice de hedge ótimo, para diferentes correlações, conforme descrito no Gráfico 5.

\section{COMENTÁRIOS FINAIS}

Quando se considera a interação entre as decisões de investimento e financiamento, aspectos de endividamento e liquidez podem influenciar o processo de criação de valor, pois têm a possibilidade de conduzir a custos ou benefícios para a empresa. Se as oportunidades de investimento são dependentes de um fator de risco que também afeta a disponibilidade de recursos internos da empresa, então a questão da gestão de riscos torna-se relevante para a otimização da riqueza do acionista.
Neste estudo, foi identificado o nível de hedge que maximiza a esperança do lucro. Através de uma avaliação analítica, obteve-se uma fórmula genérica para o nível ótimo da função de gestão de riscos. É interessante identificar como as decisões de investimento e de gestão de riscos afetam o endividamento e a liquidez quando se procura a maximização dos lucros.

Para a obtenção da sensibilidade da fórmula analítica do hedge ótimo, recorreu-se à simulação. Assim, além da contribuição teórica da incorporação de diversas decisões financeiras em um único modelo, esta pesquisa buscou, ainda, apresentar simulações que permitissem identificar inter-relacionamentos relevantes entre as variáveis.

No trabalho, a partir da definição arbitrária das funções e dos parâmetros do modelo, foram realizadas diversas simulações visando, principalmente, à análise da sensibilidade da decisão de hedge em função de flutuações de outras variáveis. Assim, relações analíticas e complexas entre variáveis puderam ser avaliadas à luz dos resultados das simulações.

A implementação prática da estratégia de hedge ótimo descrita envolve os seguintes passos: (1) a identificação do conjunto de oportunidades de investimento da empresa e o relacionamento com o fator de risco envolvido; (2) a identificação das funções de custos decorrentes do endividamento e dos benefícios que a empresa atribui à disponibilidade de recursos internos; (3) a operação de compra ou venda de contratos a termo ou futuros associados ao fator de risco e equivalentes ao índice de hedge ótimo calculado. $\mathrm{O}$ valor de referência para número ótimo de contratos é o total de recursos iniciais disponíveis que estão sujeitos à flutuação do fator de risco.

Observe, portanto, que o procedimento no mercado de derivativos envolve uma operação simples com derivativos. A contribuição do modelo está no refinamento do número de contratos a serem negociados, uma vez que a estratégia de hedge leva em consideração a correlação entre as oportunidades de investimentos e o fator de risco, além dos efeitos de necessidades de endividamento adicional e de disponibilidade de recursos internos para investimentos.

É importante destacar potenciais problemas de aplicabilidade prática da estratégia de hedge. Se o instrumento de hedge não possuir alta liquidez, o custo de transação pode diminuir consideravelmente os lucros esperados. Além disso, o derivativo utilizado para o hedge pode sofrer influências de outras variáveis e não somente do fator de risco considerado. Exemplificando, no caso de o fator de risco ser a taxa de câmbio, os instrumentos 
derivativos de câmbio disponíveis podem, também, ser sensíveis a variações nas taxas de juros, implicando um potencial risco de base na estratégia de hedge. Além disso, a instabilidade da correlação do fator de risco e das oportunidades de investimentos pode representar importante fato a ser avaliado, principalmente em cenários extremos de elevada turbulência econômica. Finalmente, questões de calibração dos parâmetros do modelo para adequação aos dados de mercado e outros aspectos práticos como formas de tributação também podem constituir pontos de atenção no uso do modelo.

Por tratar as diversas decisões financeiras de modo integrado, estabelecendo as ligações entre investimentos, financiamento, liquidez e gestão de riscos, o modelo proposto neste trabalho tem característica robusta e pode servir de base para diversas outras investigações. Por exemplo, uma sugestão para estudos futuros refere-se a um maior detalhamento da influência da política de dividendos na gestão de riscos. O modelo deste trabalho incorpora a decisão de dividendos de forma simplista. Todos os recursos disponíveis internamente são utilizados para financiamento dos investimentos $e$, portanto, a decisão de dividendos implica a retenção completa das disponibilidades. Eventualmente, um modelo mais realista poderia envolver a questão da criação ou perda de valor decorrentes da estratégia de dividendos. Nessa situação, aspectos referentes à assimetria de informação e conflitos de interesse podem ser considerados para a incorporação de outras imperfeições de mercado.

\section{REFERÊNCIAS BIBLIOGRÁFICAS}

BERKMAN, H.; BRADBURY, M. E. Empirical evidence on the corporate use of derivatives. Financial Management 25-2, p. 513, 1996.

Magan, S. An International Comparison of Derivative Use. Financial Management 26, p.69-73, 1997.

BODNAR, G.; HAYT, G. MARSTON, R. 1995 Wharton survey of derivatives usage by US nonfinancial firms. Financial Management 25, p. 113-133, 1996.

1998 Wharton survey if financial risk management by US non financial firms. Financial Management 27, p. 70-91.

CAMPBELL, T. S.; KRACAW, W. A. Optimal managerial incentive contracts and the value of corporate insurance. Journal of Financial and Quantitative Analysis 22, p. 315-328, 1987.

COHEN, R. B.; HALL, B. J.; VICEIRA, L. M. Do executive stock options encourage risktaking? Working paper, Havard Business School, 2000.

COPELAND, T.; COPELAND, M. Managing corporate FX risk: a value maximizing approach. Financial Management 28, p. 68-75, 1999.
CULP, C. The risk management process. John Wiley \& Sons, 2001.

DEMARZO, P.; DUFFIE, D. Corporate incentives for hedging and hedge accounting. Review of Financial Studies 8, p. 743-772, 1995.

DOLDE, W. The trajectory of corporate financial risk management. Journal of Applied Corporate Finance 6, p. 33-41, 1993.

Hedging, leverage and primitive risk, Journal of Financial Engimeering, 4-2,

p. 107-121, 1995.

EDERINGTON, L. H. The hedging performance of the new futures markets. Journal of Finance 34, p. 157-170, 1979.

FRANCIS, J.; STEPHAN, J. Characteristics of hedging firms: An empirical examination. in Robert J. Schwartz and Clifford W. Smith, Jr, eds: Advanced Strategies in Financial Risk Management. New York Institute of Finance, 1990.

FROOT, K.; SCHARFSTEIN, D.; STEIN, J. Risk management: coordinating corporate investment and financing policies. Journal of Finance 48, p. 1629-1658, 1993. 
GÉCZY, C.; MINTON, B. A.; SCHRAND, C. Why firms use currency derivatives. Journal of Finance 52, p. 1323-1354, 1997.

GUAY, W.; KOTHARI, S. How much do firms hedge with derivatives. Journal of Financial Economics 70, p. 423-461, 2003.

HAULSHALTER, G. Financing policy, basis risk and corporate hedging: evidence from oil and gas producers. Journal of Finance 55 , p. $107-151,2000$.

HEANEY, J.; POITRAS, G. Estimation of the optimal hedge ratio, expected utility, and ordinary least squares regression. Journal of Futures Markets 11-5, 1991.

HOWTON, S.; PERFECT, S. Currency and interest-rate derivatives use in the US firms. Financial Management (Winter), p. 111-121, 1998.

MILLER, M.; MODIGLIANI, F. The cost of capital, corporation finance and the theory of investment. American Economic Review 53, p. 261-267, 1958.

MYERS, R. J. Estimating time-varying optimal hedge ratios on futures markets. Journal of Futures Markets 20-1, p. 73-87, 2000.

MOOSA, I. Is there a need for hedging exposure to foreign exchange risk? Applied Financial Economics. 14, p. 279, 2004. NANCE, D.; SMITH, C.; SMITHSON, C., 1993. On the determinants of corporate hedging. Journal of Finance 48, p. 267-284, 1993.
NGUYEN, H.; FAFF, R. On the determinants of derivative usage by Australian companies. Australian Journal of Management, 27, p. 1-24, 2002.

PETERSEN, M. A.; THIAGARAJAN, S. R. Risk measurement and hedging: with and without derivatives. Financial Management (Winter), p. 5-30, 2000.

ROSS, M. Corporate hedging: what, why, and how? Working paper, University of California Berkeley, 1996.

RUBINSTEIN, The Valuation of uncertain icone streams and the pricing of options. Bell Journal of Economics, 7-2, p. 407-425, 1976.

SMITH, C.; STULZ, R. The determinants of firms' hedging policies. Journal of Financial and Quantitative Analysis 20, p. 391-405, 1985.

SPANO, M. Investment, debt and risk management in a context of uncertain returns to investment. Working paper. York University, 2001.

STEIN, J. L. The simultaneous determination of spot and future prices. American Economic Review 51, p. 1012-1025, 1961.

STULZ, R. Optimal hedging policies. Journal of Financial and Quantitative Analysis 19, p. 127-140, 1984.

TUFANO, P. Who manages risk? An empirical analysis of risk management practices in the gold mining industry. Journal of Finance 51, p. 1097-1137, 1996

\section{NOTA:}

Endereço dos Autores:

Universidade Presbiteriana Mackenzie

Rua da Consolação, 930

São Paulo - SP - 01302-907 